\title{
Synthesis of Superhydrophobic Surfaces with Wenzel and Cassie-Baxter State: Experimental Evidence and Theoretical Insight
}

\author{
Xia Zhang ${ }^{1 *}$, Bei Ding ${ }^{1}$, Yifei Bian ${ }^{2 *}$, Dong Jiang ${ }^{3}$, Ivan P. Parkin ${ }^{2 *}$ \\ ${ }^{1}$ National \& Local Joint Engineering Research Center for Applied Technology of Hybrid Nanomaterials, Henan \\ University, Kaifeng 475004, P.R. China. \\ ${ }^{2}$ Department of Chemistry, University College London, 20 Gordon Street, London, WC1H 0AJ, UK. \\ ${ }^{3}$ State Key Laboratory of Solid Lubrication, Lanzhou Institute of Chemical Physics, Chinese Academy of Sciences, \\ Lanzhou 730000, PR China \\ E-mail: xia.zhang@ucl.ac.uk, yifei.bian15@ucl.ac.uk,i.p.parkin@ucl.ac.uk,
}

\begin{abstract}
We demonstrate surfactant and template free synthesis of superhydrophobic thin films by controlling surface morphology. The surface morphology evolution process was determined from time dependent studies. The relationships between the water contact angle, the sliding angle, water droplet size, and the surface microstructures was investigated. It is found that structural parameters play an important role in determining the adhesion of a droplet on superhydrophobic surfaces and the liquid-solid adhesion can be effectively manipulated through tailoring the morphology or the size of the microstructures on the surface. We provide a theoretical explanation for the Cassie-Baxter state (water rolls) and the Wenzel state (sticky surface, water adheres) with droplet size on different microstructure surfaces. The new theoretical insight provided in this study match well with experimental results.
\end{abstract}

Keywords: Superhydrophobic; Wenzel state; Cassie-Baxter state; Hierarchical structures 


\section{Introduction}

Wettability is one of the most important characteristics of solid surfaces. Superhydrophobic surfaces have extreme wettability and have received much attention due to applications in self-cleaning, ${ }^{1-2}$ anti-icing, ${ }^{3-4}$ drag-reduction, ${ }^{5}$ and oil-water separation. ${ }^{6}$ Superhydrophobic surfaces are of two types - Cassie Baxter type and the Wenzel type. In the Cassie -Baxter surface water droplets often sit on the surface protrusions and easily roll across the surface. In a Wenzel surface, the water droplet penetrates the surface porosity and is pinned in place. Variation between Cassie Baxter and Wenzel behavior is known but not completely understood. In nature, the lotus leaf is a typical representative of the Cassie Baxter superhydrophobic surface and water droplets on these surfaces can roll away easily and remove dust particles, which gives rise to the self-cleaning phenomenon. ${ }^{8}$ Whereas, highly adhesive superhydrophobic surfaces are demonstrated in nature by rose petals and gecko feet. ${ }^{9}$ It is thought that the volume of water droplet may play an important role as to whether a surface displays Wenzel or Cassie-Baxter type superhydrophobicity. ${ }^{10}$ For example, on superhydrophobic materials used for rain proofing, it is found that not all water droplets roll effectively to clean the surface. Droplets below a particular size often stick even when vertical or upside down, showing a Wenzel type behavior. ${ }^{11}$ It is necessary therefore to investigate the relationships between the size of different droplets, the sliding angle, the contact angle, and the microstructure of rough hydrophobic surfaces. At present, there are a lot of reports about the rolling and adhesion of water droplets, ${ }^{12-18}$ however, a comprehensive understanding of the sliding properties of water droplets with different sizes, especially for small water droplets on superhydrophobic surfaces is still insufficient. ${ }^{19-22}$

In this study, we fabricated superhydrophobic $\mathrm{Co}_{3} \mathrm{O}_{4}$ films with different topographies by combining both simple solution-immersion process and self-assembly of stearic acid. By adjusting the reaction time, the microstructure of the $\mathrm{Co}_{3} \mathrm{O}_{4}$ films can be tailored from nanowire arrays to nanowire/microflowers structures, and finally to microflowers. We also investigate the wetting of 
Cassie-Baxter or Wenzel State for water droplets of varying volume and their relation with surface microstructures. The variation of sliding angle with different droplet volumes was modeled semi-quantitatively and the results correlated well with the experiment. That the results reported here provide an understanding of how surface roughness and water droplet size contribute to the transition wetting behavior from the Cassie-Baxter to the Wenzel State.

\section{Experimental Section}

\subsection{Preparation of superhydrophobic $\mathrm{Co}_{3} \mathrm{O}_{4}$ film}

In a typical synthesis, $5 \mathrm{mmol}$ of cobalt nitrate hexahydrate $\left(\mathrm{Co}\left(\mathrm{NO}_{3}\right)_{2} \cdot 6 \mathrm{H}_{2} \mathrm{O}\right)$ and $15 \mathrm{mmol}$ of urea $\left(\mathrm{CO}\left(\mathrm{NH}_{2}\right)_{2}\right)$ were dissolved in $50 \mathrm{~mL}$ of distilled water to form a homogeneous solution. Then a piece of clean aluminium sheet $(10 \times 30 \times 1 \mathrm{~mm})$ was immersed into the reaction solution and maintained at $97^{\circ} \mathrm{C}$ for a certain time. After that, the sample was taken out and rinsed with distilled water several times in order to remove the free particle debris and the residual reactant. The sample was annealed at $250^{\circ} \mathrm{C}$ in air for $2 \mathrm{~h}$ leading to the formation of $\mathrm{Co}_{3} \mathrm{O}_{4}$ film with controlled morphology. Finally, the obtained sample was immersed into $0.015 \mathrm{~mol} / \mathrm{L}$ of stearic acid in ethanol for in $2 \mathrm{~h}$, and taken out, washed with ethanol, and dried at $85^{\circ} \mathrm{C}$ for $1 \mathrm{~h}$.

\subsection{Characterization}

The morphologies and structures of samples were characterized by X-ray diffraction (XRD, RIGAKU D/Max-2550 with $\mathrm{Cu}-\mathrm{K} \alpha$ radiation), field emission scanning electron microscopy (JEOL JSM-6701F FESEM) and high-resolution transmission electron microscopy (HRTEM, JEOL JEM-2010F). X-ray photoelectron spectroscopy (XPS) data was acquired using a VGESCALAB210 X-ray photoelectron spectrometer. Water contact angle and sliding angle were measured using a KRUSS DSA 100 (KRuSS) apparatus. The average contact angle and sliding angle values were obtained by measuring the same sample in at least five different positions, and images were captured with a digital camera (Canon).

\section{Results and discussion}




\subsection{Structural characterization and growth of crystalline $\mathrm{Co}_{3} \mathrm{O}_{4}$ film}

Cobalt oxide films were grown on an aluminium sheet by reaction of cobalt nitrate and urea at $97^{\circ} \mathrm{C}$. The films evolved different microstructures depending on synthesis time. Fig. 1a and $\mathrm{b}$ shows the FESEM images of the sample surface formed from reaction of cobalt nitrate hexahydrate and urea after $13 \mathrm{~h}$ of reaction time. It can be seen that the surface is uniformly and densely covered with nanowires, which are joined together at top to form arches. The detailed microstructures of the nanowires were further studied by TEM. As shown in Fig. 1c, the branch is composed of 10-15 nm nanocrystals that are packed into a compact linear structure. The selected area electron diffraction (SAED) pattern is shown as an inset in Fig. 1c, and indicates that the nanowires consist of multiple crystallites. The structure and phase of the sample after annealing at $250^{\circ} \mathrm{C}$ for $2 \mathrm{~h}$ were determined by X-ray diffraction analysis (Fig. 1d). All the diffraction peaks are attributed to the spinel $\mathrm{Co}_{3} \mathrm{O}_{4}$ phase (JCPDS card No. 42-1467) indicating that crystalline $\mathrm{Co}_{3} \mathrm{O}_{4}$ has been successfully formed after annealing. The cubic spinel phase of $\mathrm{Co}_{3} \mathrm{O}_{4}$ was found to have a lattice constant of $\mathrm{a}=8.084 \AA$. This is also consistant with standard XRD pattern for $\mathrm{Co}_{3} \mathrm{O}_{4}$ (JCPDS card No. 42-1467, a = 8.084 ). No impurity peaks were detected by XRD, which showed that the cobalt oxide contained no easily measurable second crystalline phase.

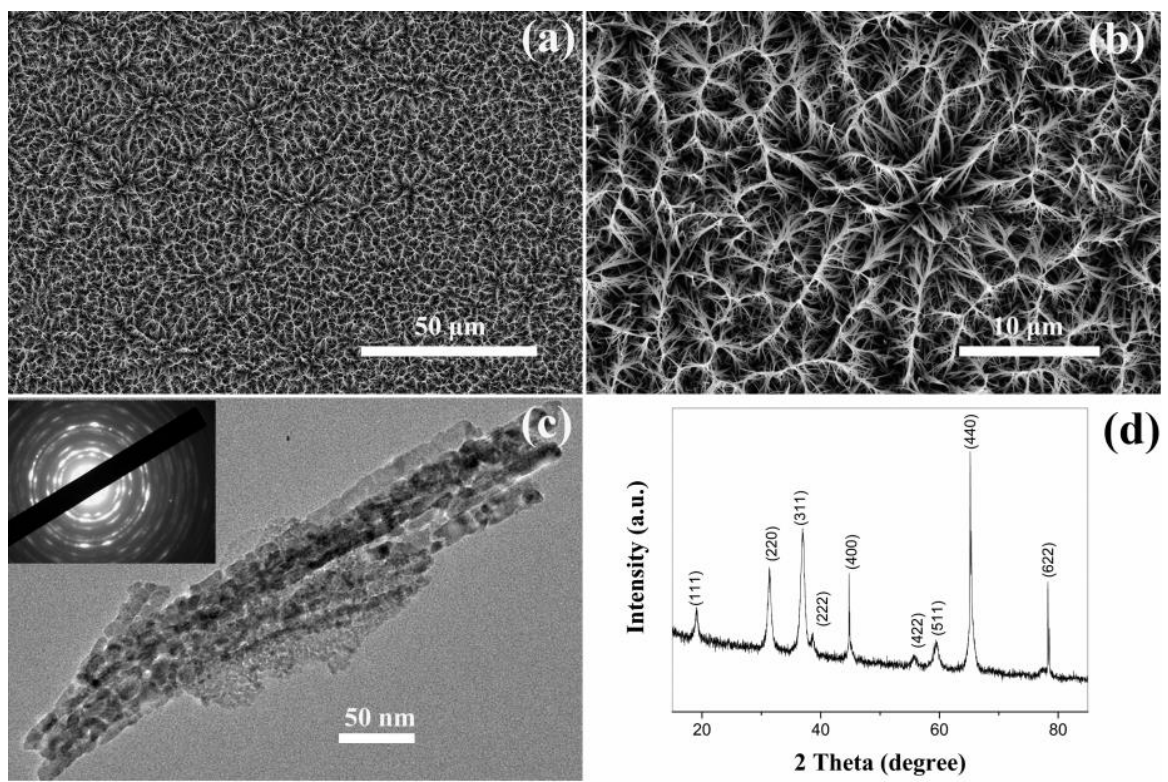


Fig. 1 Characterization of the of the $\mathrm{Co}_{3} \mathrm{O}_{4}$ film with $13 \mathrm{~h}$ immersion process and after annealing at $250^{\circ} \mathrm{C}$ for $2 \mathrm{~h}$. (a, b) SEM images; (c) TEM image; (d) XRD pattern. Inset in (c) is the corresponding SAED pattern.

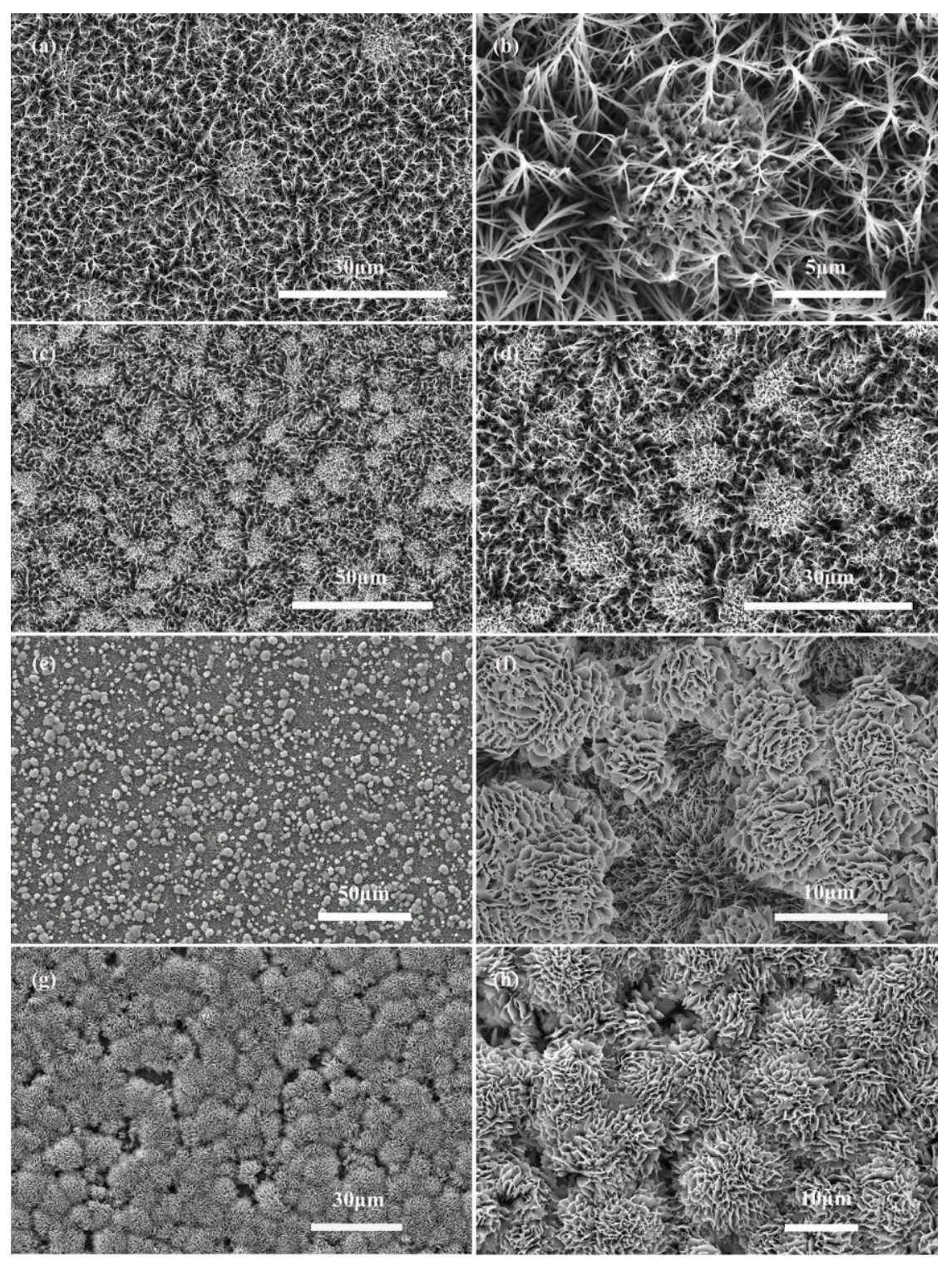

Fig. 2 SEM images of the film with different reaction time. (a, b) 15 h; (c, d) 16 h; (e, f) 18 h; (e, f) $20 \mathrm{~h}$.

By adjusting the reaction time, the morphology of the $\mathrm{Co}_{3} \mathrm{O}_{4}$ film was tailored to different microstructures, as shown in Fig. 2. When the immersion time reaches $15 \mathrm{~h}$, a few nanosheets grow from the nanowires arrays and this is the genesis of the initial formation of the microflower, as shown in Fig. 2a and b. A further increase in the immersion time leads to an increased surface 
area of the microflower structures and the microflowers coexist with nanowires on the surface, showing the microflower/nanowire array structures (Fig. 2c, d). When the immersion time reaches $18 \mathrm{~h}$, an increasing number of microflowers appear and the microflowers grow bigger and of very high density. The high-resolution SEM image (Fig. 2f) reveals that the obtained structure is composed of two parts. The upper part contains microflowers and the bottom is made up of oriented nanowire arrays. The microflowers are in fact hierarchical architectures consisting of nanosheets as building units which exhibit open structures with numerous nanosheets that extended outside and seem to be core gradually compacted inside. Further increase in the reaction time to $20 \mathrm{~h}$ allowed numerous microflowers with diameters of 8-10 $\mu \mathrm{m}$ that completely covered the nanowires structure, as shown in Fig. $2 \mathrm{~g}$, h. Close observation reveals that the hierarchical microflowers contain dozens of nanopetals about $0.8-1.5 \mu \mathrm{m}$ wide and $10-20 \mathrm{~nm}$ thick.

From the time-dependent morphology evolution processes, it is generally believed that the growth of the hierarchical architecture undergoes three stages: (i) formation and growth of nanowires; (ii) formation and growth of flower-like structures from nanowire arrays; (iii) further growth of microflowers on top of the nanowire array films. The growth process is shown in Fig. 3, and the chemical reaction equations for the formation of cobalt oxide film are suggested as follows: ${ }^{23}$

$\mathrm{CO}\left(\mathrm{NH}_{2}\right)_{2}+\mathrm{H}_{2} \mathrm{O} \rightarrow 2 \mathrm{NH}_{3}+\mathrm{CO}_{2}$

$\mathrm{NH}_{3}+\mathrm{H}_{2} \mathrm{O} \rightarrow \mathrm{NH}_{4}^{+}+\mathrm{OH}^{-}$

$\mathrm{CO}_{2}+\mathrm{H}_{2} \mathrm{O} \rightarrow 2 \mathrm{H}^{+}+\mathrm{CO}_{3}^{2-}$

$2 \mathrm{Co}^{2+}+2 \mathrm{OH}^{-}+\mathrm{CO}_{3}^{2-}+\mathrm{H}_{2} \mathrm{O} \rightarrow \mathrm{Co}_{2}(\mathrm{OH})_{2} \mathrm{CO}_{3} \cdot \mathrm{H}_{2} \mathrm{O}$

$3 \mathrm{Co}_{2}(\mathrm{OH})_{2} \mathrm{CO}_{3} \cdot \mathrm{H}_{2} \mathrm{O}+\mathrm{O}_{2} \rightarrow 2 \mathrm{Co}_{3} \mathrm{O}_{4}+6 \mathrm{H}_{2} \mathrm{O}+3 \mathrm{CO}_{2}$ (calcination)

Since no organic additives such as surfactants or templates existed in the reaction, the concentrations of $\mathrm{Co}^{2+}$ and $\mathrm{OH}^{-}$were likely to play the key roles. Therefore, it may be more convincing to explain the process by employing an oriented attachment crystal growth model. At 
the initial reaction stage, accompanying the $\mathrm{CO}_{3}{ }^{2-}$ and $\mathrm{OH}^{-}$anions from hydrolysis of urea reacting with $\mathrm{Co}^{2+}$ cations, the initial crystal nucleus of cobalt carbonate hydroxide hydrate start to come into being (Supporting Information Fig. S1). As the freshly formed nano-nuclei are thermodynamically unstable due to their high surface energy, they tend to gather together to minimize the interfacial energy, and thus, the supersaturated nuclei aggregate together and form nanowire arrays. During subsequent stages, cobalt carbonate hydroxide hydrate nanowires grow further in length and width into nanosheets which coexist with the nanowires. With an increase in reaction time, more and more nanosheets appear, grow larger and form hierarchical microflower architectures by consuming core materials via an Ostwald ripening process. Finally, hierarchical microflowers consisting of nanosheets as building units cover almost completely the nanowire structures. After calcination at each stage, we found that the morphologies unchanged, and the morphology evolution of cobalt carbonate hydroxide hydrate was shown in Supporting Information (Fig. S2-S6). Hence, it is believed that the cobalt carbonate hydroxide hydrate film transforms into $\mathrm{Co}_{3} \mathrm{O}_{4}$ film and the microstructures to all intense and purposes remain the same a topotactic process. ${ }^{24}$

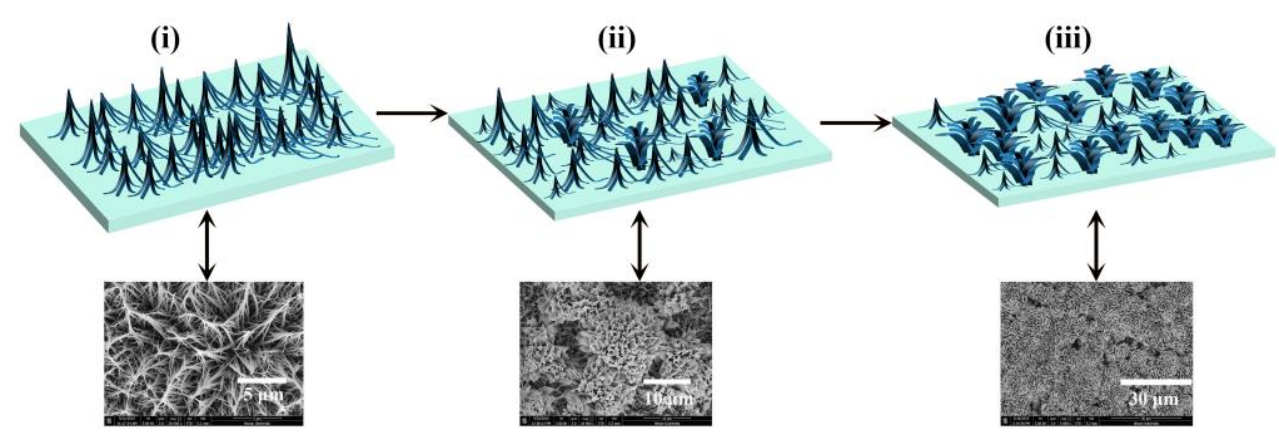

Fig. 3 Schematic of the microstructure evolution process of $\mathrm{Co}_{3} \mathrm{O}_{4}$ film and SEM image is corresponding to each growth stage (i, ii and iii represent the reaction time corresponding to $13 \mathrm{~h}$, $18 \mathrm{~h}, 20 \mathrm{~h}$ respectively).

In order to further understand the composition and the wettability transition from superhydrophilic to superhydrophobicity after chemical modification with stearic acid, XPS 
measurements were taken. Fig. $4 \mathrm{a}$ gives the survey spectrum of the as-prepared $\mathrm{Co}_{3} \mathrm{O}_{4}$ film before chemical modification, and only elements $\mathrm{Co}, \mathrm{O}$ (and adventitious $\mathrm{C}$ ) were detected, indicating the surface purity of the product. As shown in the inset in Fig. $4 \mathrm{a}$, two peaks at $780.1 \mathrm{eV}\left(\mathrm{Co} 2 \mathrm{p}_{3 / 2}\right)$ and $795.0\left(\mathrm{Co} 2 \mathrm{p}_{1 / 2}\right)$ with a spin-orbit splitting of $14.9 \mathrm{eV}$ strictly verify the formation of $\mathrm{Co}_{3} \mathrm{O}_{4}{ }^{25}$ Compared with that of before chemical modification with stearic acid, the $\mathrm{C}$ content increases and the increasing $\mathrm{C}$ content comes from the surface attached stearic acid (Fig. 4b). The $\mathrm{O} 1 \mathrm{~s}$ spectra (inset in Fig. 4b) can be modeled to resolved into multiple components centered at binding energy of $530.5,531.3$ and $532.0 \mathrm{eV}$, which are ascribed to $\mathrm{Co}-\mathrm{O}, \mathrm{C}=\mathrm{O}$, and $-\mathrm{OH}$ species, respectively. ${ }^{26}$ The XPS analysis indicates that very thin layers of stearic acid are attached to the surface of the $\mathrm{Co}_{3} \mathrm{O}_{4}$ film.
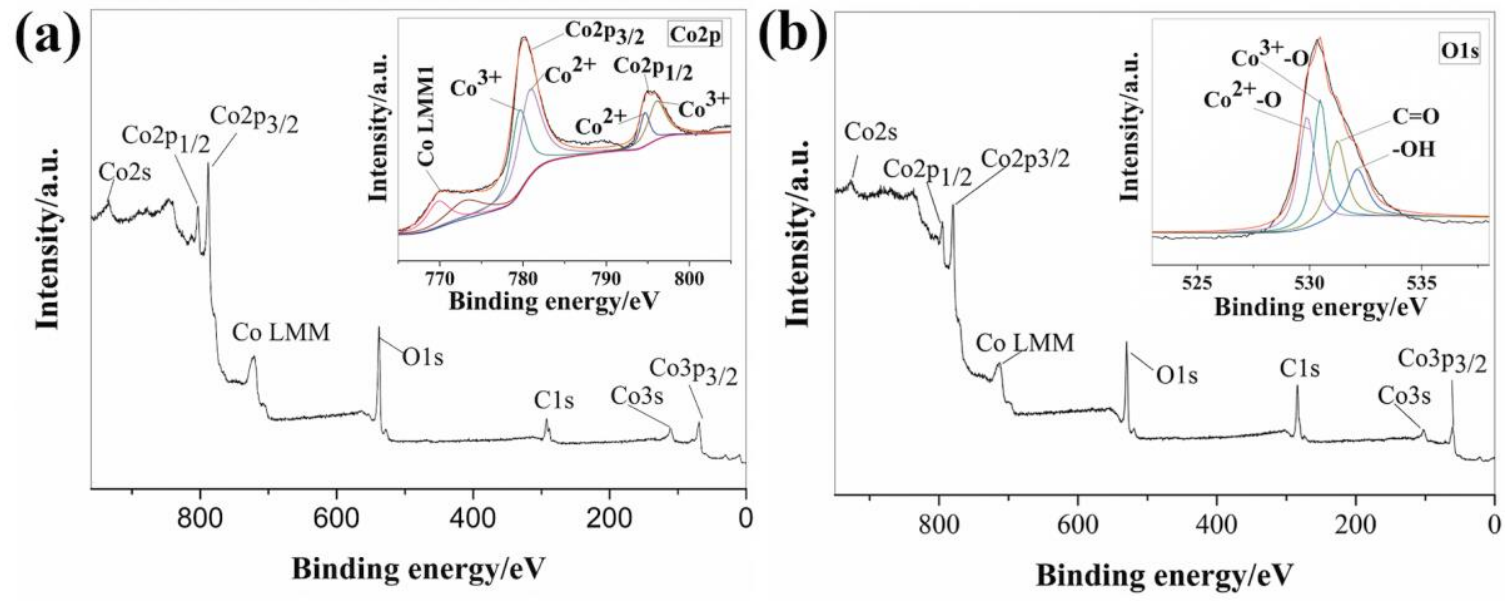

Fig. 4 XPS spectra for the obtained film surface before (a) and after (b) stearic acid modification. Inset in (a) and (b) is the decomposed spectra of Co2p and O1s, respectively.

\subsection{Morphology-Dependent Wetting Behavior}

This work shows a transition from a Wenzel to Cassie-Baxter surface with droplet volume and the surface microstructures trap sufficient air and play an important role for the contact angle and sliding angle values because it is helpful in the variation of the liquid/solid contact area. Herein, we investigate the influence of the surface microstructures and water droplet size with the contact angle and sliding angle values. Fig. 5 (a) shows the contact angle behavior of water droplets of various volumes on our surfaces with different morphology. A reduction in droplet volume from 
$10 \mu \mathrm{L}$ to $1 \mu \mathrm{L}$ leads to a change of contact angles on the same surface that do not vary within inherent errors of the measurement. The size of a water droplet from this work shows that it is not a key factor that affects the contact angle at the contact line. Hence, regardless of the size of the droplet, the contact angle should always be the same, and this result is consistent with that from the literature. ${ }^{24}$ However in our work, we found that for the droplets of equal volume the sliding angle was markedly different and was dependent on the surface morphology. Fig. 5 (b) shows the droplet volume as a function of the sliding angle values on different microstructured surfaces. It is clear that the sliding angle values increase with a reduction of the water drop volume on the surface with the same microstructure. On our nanowire array surface, the sliding angle value decreases from 20 $\pm 1^{\circ}$ at a drop volume of $1 \mu \mathrm{L}$ to $4 \pm 1^{\circ}$ at a drop volume of $10 \mu \mathrm{L}$. With increasing reaction time, the surface microstructure turns from a nanowire array to nanowire and microflower which all coexist, and finally to hierarchical microflowers. As shown in Fig. 5 (a) and (b), for a water drop of $10 \mu \mathrm{L}$, the contact angle value will decrease from $164 \pm 1^{\circ}$ on the nanowire array surface $(13 \mathrm{~h}$ reaction time) to $158 \pm 1^{\circ}$ on the microflower structure surface (18 $\mathrm{h}$ reaction time), whereas the corresponding sliding angle value will increase from $4 \pm 1^{\circ}$ to $15 \pm 1^{\circ}$.
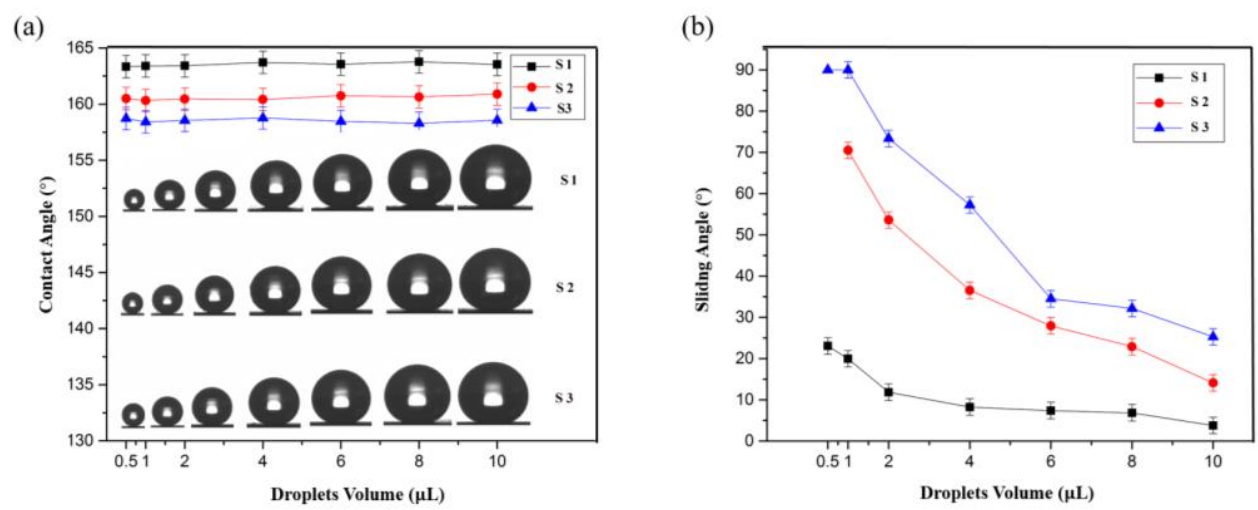

Fig. 5 The relationship between contact angle (a), sliding angle (b) value and droplet volume on different surface with the reaction time of $13 \mathrm{~h}(\mathrm{~S} 1), 16 \mathrm{~h}(\mathrm{~S} 2), 18 \mathrm{~h}$ (S3). The volume of droplets inset in (a) from left to right is $0.5,1,2,4,6,8,10 \mu \mathrm{L}$. 

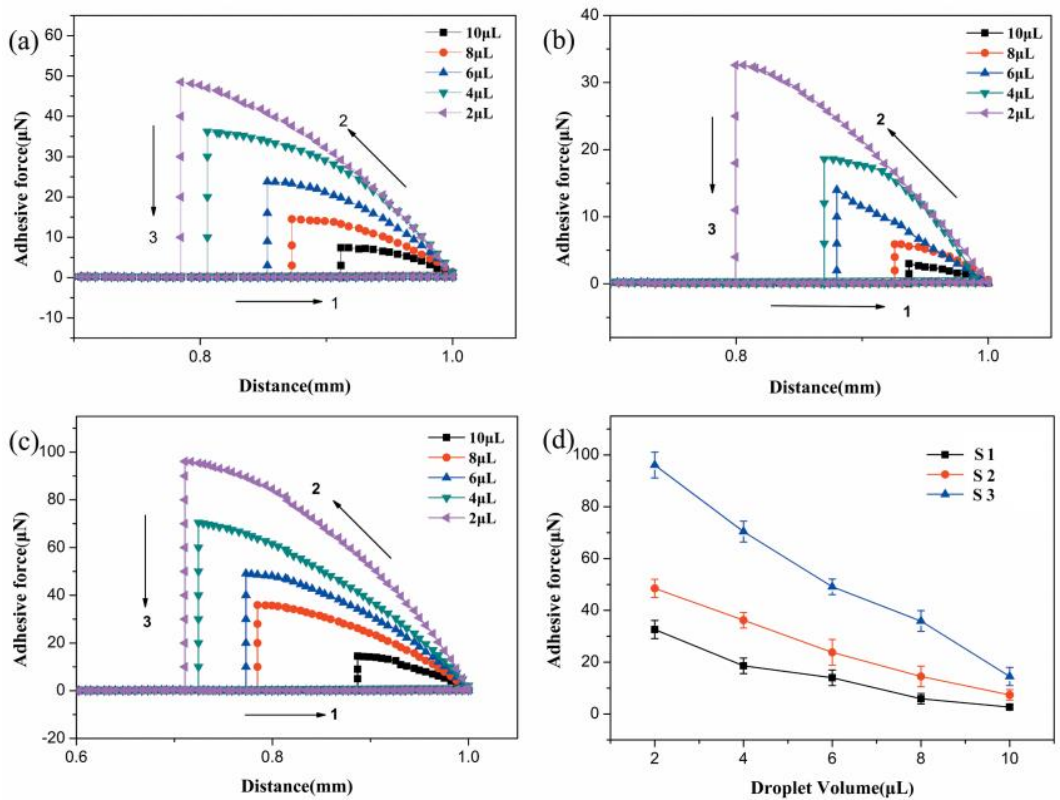

Fig. 6 Force- distance curves on the superhydrophobic surfaces under the reaction time of $13 \mathrm{~h}$ (a), $16 \mathrm{~h}(\mathrm{~b}), 18 \mathrm{~h}(\mathrm{c})$. The relationship between the adhesive force and the droplet volume (d).

The adhesive force for different droplet on the obtained superhydrophobic surface with different microstructures was conducted to further investigate the variation of sliding angle. Adhesive action was assessed by a high-sensitivity microelectromechanical balance system. First, the superhydrophobic surface was placed on the plate of the balance system, microdroplets with different volume were suspended on a metal ring, and the force of the balance system was initialized to zero. Then, the surface was brought into contact with the microdroplet while maintaining the balance force at zero (step 1). The surface was moved at a rate of $0.05 \mathrm{~mm} / \mathrm{s}$. When the surface left the microdroplet after contact, the balance force increased gradually and reached its maximum at the end of the step 2. Finally, the balance force decreased immediately when the surface broke away from the microdroplet in step 3 to finish one cycle of the measurement. Fig. 6 (a), (b) and (c) show force-distance curves on the superhydrophobic surfaces under the reaction time of $13 \mathrm{~h}, 16 \mathrm{~h}$, and $18 \mathrm{~h}$. It can be seen that the adhesive force increases with the decrease of the droplet volume. The big droplet has a smaller adhesive force, and droplet with a bigger size has a smaller adhesive force. Thus, the smaller drop is more likely to be fixed on the surface. Therefore, 
the sliding angle will decrease with droplet volume increase. The surface roughness plays an important role in the sliding angle of a droplet with a fixed size. As shown in Fig. 6d, the adhesive force of a droplet is lower on nanowire surface than on microflower structure surface, which lead to the droplet having a smaller sliding angle on nanowire surface, and a bigger one on microflower structure surface.

\subsection{Theoretical insight into wetting behavior}

The nanowire array structure surface is much rougher than that of the microflower structure surface, and typically water contact angle has a higher value on a rougher surface, and this can be verified with theory. The contact angle of a sessile droplet on a smooth solid surface is described by Young's equation:

$$
\cos \theta_{Y}=\frac{\gamma_{S V}-\gamma_{S L}}{\gamma_{L V}}
$$

where $\gamma_{S V}, \gamma_{S L}$ and $\gamma_{L V}$ are the surface free energy of solid-vapour, solid-liquid and liquid-vapour interfaces respectively. For a rough surface, the droplets' wetting behavior can be considered within two regimes. The Wenzel regime, who proposed an equation to describe homogeneous wetting, where droplets penetrate the surface roughness protrusions: $:^{27}$

$$
\cos \theta_{W}=r \cos \theta_{Y}
$$

Here $\theta_{W}$ is the equilibrium contact angle and $\mathrm{r}$ is the surface roughness, which is defined as the product of actual solid surface area to the contact area. However, the equation describing the heterogeneous wetting is given by Cassie and Baxter: ${ }^{28} \cos \theta_{C B}=r f \cos \theta_{Y}+f-1$

where $\mathrm{f}$ is the surface area fraction on the rough surface. So for hydrophobic surfaces whose $\theta_{Y}>$ $90^{\circ}$, increasing its roughness will result in an increased contact angle.

Commonly, a surface with higher contact angle will also show a lower sliding angle.

Theoretically, Furmidge ${ }^{29}$ reported his derived equation on the sliding angle:

$$
\frac{m g \cdot \sin \alpha}{w}=\gamma_{L V}\left(\cos \theta_{R}-\cos \theta_{A}\right)
$$


where $\mathrm{m}$ is the mass of droplet, $\mathrm{g}$ is the gravitational constant, $\alpha$ is the $\mathrm{SA}, \mathrm{w}$ is the width of droplet, $\theta_{R}$ and $\theta_{A}$ are the receding and advancing contact angle s of droplet. The right-hand side of the equation is dependent on the properties of the surface and inside the parenthesis is the contact angle hysteresis.

The droplets during this experiment are smaller than $10 \mu \mathrm{L}$, which can be approximated as a spherical cap when stationary on the substrate, whose volume is given by

$$
V=\frac{\pi R^{3}(1-\cos \theta)^{2}(2+\cos \theta)}{3 \sin ^{3} \theta}
$$

Then the contact radius

$$
\mathrm{R}=\left(\frac{3 \sin ^{3} \theta \cdot V}{\pi(1-\cos \theta)^{2}(2+\cos \theta)}\right)^{\frac{1}{3}}
$$

Here the width of droplet is assumed as $\mathrm{w}=2 \mathrm{R}$. Thus, for substrates with the same surface condition, droplets above should show the relationship

$$
\sin \alpha \propto \frac{R}{m} \equiv f_{F}
$$

The equation is similar to what Wolfram et al reported ${ }^{30}$

$$
\sin \alpha=\frac{2 k R \pi}{m g}
$$

Where $\mathrm{k}$ is a constant related to the interaction energy between liquid and solid surface. As they claimed that this empirical equation is only valid for smooth surfaces, Masashi Miwa and his co-workers investigated the relationship between contact angle and sliding angle on a rough surface, modifying eq. 8 with several assumptions: ${ }^{31}$

$$
\sin \alpha=\frac{2 R k \sin \theta(1+\cos \theta)}{g\left(1+R \cos \theta^{\prime}\right)}\left[\frac{3 \pi^{2}}{m^{2} \rho\left(2-3 \cos \theta+\cos ^{3} \theta\right)}\right]^{1 / 3}
$$

Here $\theta$ is and $\theta^{\prime}$ are the contact angle of the rough and flat surfaces. Therefore, droplets on the same substrate would illustrate the relationship

$$
\sin \alpha \propto \frac{R}{\left(1+R \cos \theta^{\prime}\right) m^{\frac{2}{3}}}
$$


For droplets with volume $<10 \mu \mathrm{L}$ and contact angle $>150^{\circ}$, the contact radius $\mathrm{R}$ is of order $10^{-4}$ $\mathrm{m}$, thus $1+R \cos \theta^{\prime} \approx 1$, then eq. 10 can be simplified to

$$
\sin \alpha \propto \frac{1}{m^{\frac{1}{3}}} \equiv f_{M}
$$

Following eq. 6 the range of contact radius of the droplets used in our experiments is calculated to be $130 \mu m \leq R \leq 490 \mu m$, which is far larger than the scale of the micro-structures shown in SEM image (Fig. 2). Thus it is considered that the surface nano-structure transforms from nanowire arrays to microflowers as the immersion times increase, which slightly lowers the surface roughness and then leads to the reduction of the water contact angle.

In line with eq. 11 , as $m=\rho V$, the curves $\sin \alpha-\frac{1}{V^{\frac{1}{3}}}$ are plotted, as shown in Fig. 7 , the curves with reaction time of $13 \mathrm{~h}$ and $16 \mathrm{~h}$ show an approximate linear tendency. For the immersion time of $18 \mathrm{~h}$, the curve is not that consistent with eq. 11 , it is considered that the wetting is partly in the Wenzel regime while the relationship of eq.11 is based on the Cassie-Baxter model. This also explains when the droplet volume is $0.5 \mu \mathrm{L}$, the sliding angle reaches $90^{\circ}$ as the droplets stick to the surface.

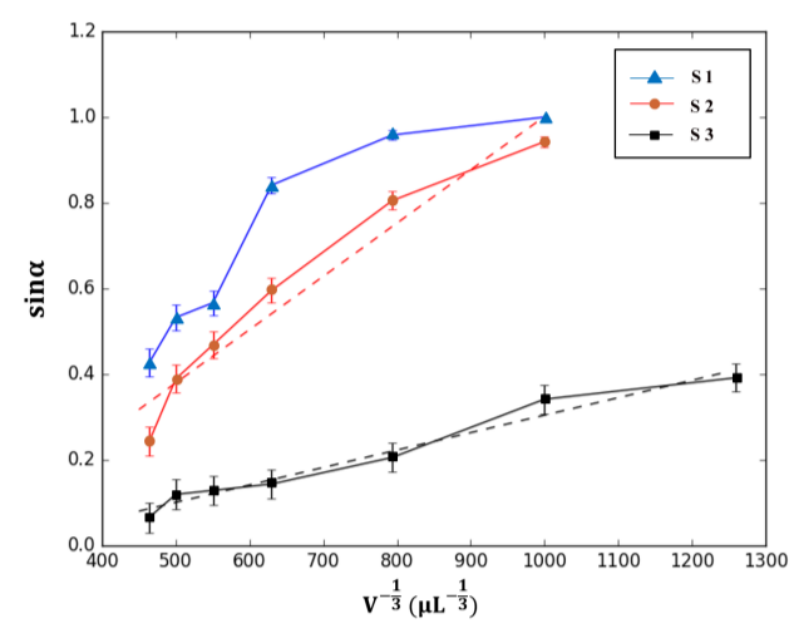

Fig. 7 Sine value of the sliding angle plotted against $\mathrm{V}^{-\frac{1}{3}}, \boldsymbol{\Delta}, \bullet$ and $\boldsymbol{\square}$ represent actual data points, and the dotted lines equal the theoretical calculation.

\section{Conclusions}


In conclusion, we have fabricated $\mathrm{Co}_{3} \mathrm{O}_{4}$ superhydrophobic films with controlled microstructures through a simple immersion chemistry approach. With increased reaction time, the microstructures can be tailored from nanowire arrays to three-dimensional hierarchical microflowers consisting of nanosheets as building units. Water droplets with different volumes have different contact angle and sliding angle values on the obtained films, and when compared with the microflowers structure surface, the water contact angle on nanowire array structure surface has a high value. The variation trend of contact angle on $\mathrm{Co}_{3} \mathrm{O}_{4}$ surface with an increasing reaction time is believed to be due to a reduction in surface roughness during the microstructure transformation, from high-roughness nanowire to low-roughness hierarchical microflower surface. Meanwhile, the experimental data of sliding angle with different droplet volumes compares well with the derived $\sin \alpha-\frac{1}{\mathrm{~V}^{\frac{1}{3}}}$ relationship, except for very small droplets in the Wenzel wetting regime.

\section{Acknowledgements}

This work was supported by International Exchange Scheme- China NSFC/U.K. Royal Society (21711530209) and the National Nature Science Foundation of China (Grant No. 51505463, 21403055). Ivan P. Parkin acknowledges the EPSRC M3S CDT grant (EP/L015862/1).217

\section{References}

(1) Wu, Y. Q.; Jia, S. S.; Qing, Y.; Luo, S.; Liu, M. A. A Versatile and Efficient Method to Fabricate Durable Superhydrophobic Surfaces on Wood, Lignocellulosic Fiber, Glass, and Metal Substrates. J. Mater. Chem. A, 2016, 4, 14111-14121.

(2) Peng, Y. B.; Guo, Z. G. Recent Advances in Biomimetic Thin Membranes Applied in Emulsified Oil/Water Separation. J. Mater. Chem. A, 2016, 4, 15749-15770.

(3) Weng, C. J.; Chang, C. H.; Peng, C. W.; Chen, S. W.; Yeh, J. M.; Hsu, C. L.; Wei, Y. Advanced Anticorrosive Coatings Prepared from the Mimicked Xanthosoma Sagittifolium-leaf-like Electroactive Epoxy with Synergistic Effects of Superhydrophobicity and Redox Catalytic Capability. Chem. Mater. 2011, 23, 2075-2083. 
(4) Kulinich, S. A.; Farhadi, S.; Nose, K.; Du, X. W. Superhydrophobic Surfaces: Are They Really Ice-Repellent? Langmuir, 2010, 27, 25-29.

(5) Zhang, X. Y.; Li, Z.; Liu, K. S.; Jiang, L. Bioinspired Multifunctional Foam with Self-Cleaning and Oil/Water Separation. Adv. Funct. Mater. 2013, 23, 2881-2886.

(6) Lu, Y.; Sathasivam, S.; Song, J. L.; Chen, F.; Xu, W. J.; Carmalt, C. J.; Parkin, I. P. Creating Superhydrophobic Mild Steel Surfaces for Water Proofing and Oil-Water Separation. J. Mater. Chem. A. 2014, 2, 11628-11634.

(7) Li, J.; Jing, Z. j.; Yang, Y. X.; Wang, Q. T.; Lei, Z. Q. From Cassie State to Gecko State: a Facile Hydrothermal Process for the Fabrication of Superhydrophobic Surfaces with Controlled Sliding Angles on Zinc Substrates. Surface and Coatings Technology, 2014, 258, 973-978.

(8) Crick, C. R.; Parkin, I. P. Relationship Between Surface Hydrophobicity and Water Bounces - a Dynamic Method for Accessing Surface Hydrophobicity. J. Mater. Chem. A. 2013, 1, 799-804.

(9) Feng, L.; Zhang, Y. N.; Xi, J. M.; Zhu, Y.; Wang, N.; Xia, F.; Jiang L. Petal Effect: a Superhydrophobic State with High Adhesive Force. Langmuir, 2008, 24, 4114-4119.

(10) Liu, M. J.; Lei J., Insitu Surface Reaction Induced Adhesion Force Change for Mobility Control, Droplet Sorting and Bio-Detection. Adv. Funct. Mater. 2010, 20, 3753-3764.

(11)Liu, X. J.; Liu, Z. L.; Liang, Y. M.; Zhou, F. In Situ Surface Reaction Induced Adhesion Force Change for Mobility Control, Droplet Sorting and Bio-Detection. Soft Matter. 2012, 8, 10370-10377.

(12) Soe, K.; Kim, M.; Ahn, J. K.; Kim, D. H. Effects of Drop Size and Measuring Condition on Static Contact Angle Measurement on a Superhydrophobic Surface with Goniometric Technique. Korean J. Chem. Eng. 2015, 32, 2394-2399.

(13)Wu, D.; Wu S. Z.; Chen, Q. D.; Zhang, Y. L.; Yao, J.; Yao, X.; Niu, L. G.; Wang, J. N.; Jiang, L.; Sun, H. B. Curvature-Driven Reversible In Situ Switching Between Pinned and 
Roll-Down Superhydrophobic States for Water Droplet Transportation. Adv. Mater. 2011, 23, $545-549$.

(14)Wang, D A.; Liu Y.; Liu X. J.; Zhou F.; Liu W. M. Towards a Tunable and Switchable Water Adhesion on a $\mathrm{TiO}_{2}$ Nanotube Film with Patterned Wettability. Chem. Commun. 2009, 45, $7018-7020$.

(15) Tian, D. L.; Chen, Q. W.; Nie, F. Q.; Xu, J. J; Song, Y. L; Jiang, L. Patterned Wettability Transition by Photoelectric Cooperative and Anisotropic Wetting for Liquid Reprography. Adv. Mater. 2009, 21, 3744.

(16) Li, J.; Liu, X. H.; Ye, Y. P.; Zhou, H. D.; Chen, J. M. Fabrication of Superhydrophobic CuO Surfaces with Tunable Water Adhesion. J. Phys. Chem. C 2011, 110, 4726-4729.

(17) Yin, X. Y.; Wang, D. A.; Liu, Y. P.; Yu, B.; Zhou, F. Controlling Liquid Movement on a Surface with a Macro-Gradient Structure and Wetting Behavior. J. Mater. Chem. A. 2014, 2, $5620-5624$.

(18)Li, C.; Guo, R. W.; Jiang, X.; Hu, S. X.; Li, L.; Cao, X. Y. Yang, H.; Song, Y. L.; Ma, Y. M.; Jiang, L. Reversible Switching of Water-Droplet Mobility on a Superhydrophobic Surface Based on a Phase Transition of a Side-Chain Liquid-Crystal Polymer. Adv. Mater. 2009, 21, 4254-4258.

(19)Liu, X.; Ye, Q.; Yu, B.; Liang, Y.; Liu, W.; Zhou, F. Switching Water Droplet Adhesion Using Responsive Polymer Brushes. Langmuir, 2010, 26, 12377-12382.

(20) Su, Y. W.; Ji, B. H.; Huang, Y. G.; Hwang, K. C. Nature's Design of Hierarchical Superhydrophobic Surfaces of a Water Strider for Low Adhesion and Low-Energy Dissipation. Langmuir, 2010, 26, 18926-18937.

(21) Miwa, M.; Nakajima, A.; Fujishima, A.; Hashimoto, K.; Watanabe, T. Effects of the Surface Roughness on Sliding Angles of Water Droplets on Superhydrophobic Surfaces. Langmuir, 2000, 16, 5754-5760. 
(22) Lopes, D. M.; S. Ramos, M. M.; de Oliveira, L. R.; Mombach, J. C. M. Cassie-Baxter to Wenzel State Wetting Transition: a 2D Numerical Simulation. RSC Adv. 2013, 3, 2453024534.

(23) Zhang, X.; Guo, Y. G.; Liu, Y.; Yang, X.; Pan, J. Q.; Zhang, P. Y. Facile Fabrication of Superhydrophobic Surface with Nanowire Structures on Nickel Foil. Applied Surface Science. 2013, 287, 299-303.

(24) Lei, Y.; Li, J.; Wang, Y. Y.; Gu, L.; Chang, Y. F.; Yuan, H. Y.; Xiao, D. Rapid Microwave-Assisted Green Synthesis of 3D Hierarchical Flower-Shaped $\mathrm{NiCo}_{2} \mathrm{O}_{4}$ Microsphere for High-Performance Supercapacitor. ACS Appl. Mater. Interfaces, 2014, 6, $1773-1780$.

(25) Ge, D. H.; Geng, H. B.; Wang, J. Q.; Zheng, J. W.; Pan, Y.; Cao, X. Q.; Gu, H. W. Rapid and Large-scale Synthesis of Bare $\mathrm{Co}_{3} \mathrm{O}_{4}$ Porous Nanostructures From an Oleate Precursor as Superior Li-ion Anodes with Long-cycle Lives. Nanoscale, 2016, 45, 13509-13513.

(26) Seo, K.; Kim, M.; Ahn, J. K.; Kim, D. H. Effects of Drop Size and Measuring Condition on Static Contact Angle Measurement on a Superhydrophobic Surface with Goniometric Technique. Chem. Eng. 2015, 32, 2394-2399.

(27) Wenzel, R. N. Surface Roughness and Contact Angle. J. Phys. Colloid Chem. 1949, 53, $1466-1467$.

(28) Cassie, A. B. D. Discuss Contact Angles. Discuss. Faraday Soc. 1948, 3, 11-16.

(29) Furmidge, C. G. L. The Sliding of Liquid Drops on Solid Surfaces and a Theory for Spray Retention. Journal of Colloid Science. 1962, 17, 309-324.

(30) Wolfram, E.; Faust, R.; Padday, Ed. J. F. Wetting, Spreading, and Adhesion. Academic Press: London, 1978, Chapter 10. 
(31) Miwa, M.; Nakajima, A.; Fujishima, A.; Hashimoto, K.; Watanabe,T. Effects of the Surface Roughness on Sliding Angles of Water Droplets on Superhydrophobic Surfaces. Langmuir. 2000, 16, 5754-5760. 